\title{
Continuous quantum measurement of a light-matter system
}

\author{
R. Zhao, ${ }^{1}$ S. D. Jenkins, ${ }^{1}$ C. J. Campbell, ${ }^{1}$ D. N. Matsukevich, ${ }^{2}$ T. Chanelière, ${ }^{3}$ T. A. B. Kennedy, ${ }^{1}$ and A. Kuzmich ${ }^{1}$ \\ ${ }^{1}$ School of Physics, Georgia Institute of Technology, Atlanta, Georgia 30332-0430, USA \\ ${ }^{2}$ JQI and Department of Physics, University of Maryland, College Park, Maryland 20742, USA \\ ${ }^{3}$ Laboratoire Aimé Cotton, CNRS-UPR 3321, Bâtiment 505, Campus Universitaire, F-91405 Orsay Cedex, France
}

(Received 24 December 2009; published 11 March 2010)

\begin{abstract}
Continuous measurements on correlated quantum systems, in addition to providing information on the state vector of the system in question, induce evolution in the unmeasured degrees of freedom conditioned on the measurement outcome. However, experimentally accessing these nontrivial regimes requires high-efficiency measurements over time scales much longer than the temporal resolution of the measurement apparatus. We report the observation of such a continuous conditioned evolution in the state of a light-collective atomic excitation system undergoing photoelectric measurement.
\end{abstract}

DOI: 10.1103/PhysRevA.81.031802

PACS number(s): 42.50.Dv, 03.65.Ud, 03.67.Mn

Continuous measurements provide information on the conditional evolution of the state of a quantum system (see, e.g, Refs. [1,2]), but examples of such measurements are rare in the literature. It has been observed that photoelectric detection of a field emitted from an optical cavity causes abrupt changes in the state of the intracavity field [3-5]. The quantum theory of continuous measurements also predicts dissipative conditional evolution during periods in which no detection events are registered. However, for such a conditioned evolution to be observed, the detection apparatus must have high efficiency. Otherwise, a lack of detection cannot be interpreted as the absence of a field excitation, and, hence, one obtains little information about the underlying state. Therefore, observation of the complete conditional dynamics is difficult. Conditional dynamics has been observed, however, in a superconducting qubit system [6].

The goal is to continuously monitor, with high efficiency and temporal resolution, a system with significant quantum correlations. It is often difficult to meet this challenge in experimentally accessible systems. For example, parametric down-conversion has been a successful paradigm for a variety of investigations involving correlated quantum states [7]. It does not, however, readily lend itself to continuous quantum measurement. To understand this, consider a laser pulse incident on a nonlinear crystal. The nonlinear interaction yields a state for the down-converted fields of the form $|\psi\rangle \sim \mid$ vac $\rangle+$ $\chi \int d t d t^{\prime} f\left(t, t^{\prime}\right) \hat{\psi}_{s}^{\dagger}(t) \hat{\psi}_{i}^{\dagger}\left(t^{\prime}\right)|\mathrm{vac}\rangle+O\left(\chi^{2}\right)$, where the interaction strength $\chi \ll 1, \hat{\psi}_{s}^{\dagger}(t)$ and $\hat{\psi}_{i}^{\dagger}\left(t^{\prime}\right)$ are continuous boson operators for the signal and idler fields, respectively, and the two-photon amplitude $f\left(t, t^{\prime}\right)$ is, in general, a nonseparable function of $t$ and $t^{\prime}$. This nonseparability is often referred to as time-frequency entanglement and has an associated time scale much shorter than photodetector resolution times [8,9]. The degree of spectral filtering required would result in an unacceptably low detection efficiency, which as we note below, makes conditional evolution unobservable.

A cold atomic ensemble can provide a system analogous to parametric amplification while ensuring that the joint signal-idler amplitude remains separable. In this Rapid Communiation, we consider an ensemble of $N \gg 1$ atoms in a $\Lambda$ level configuration with ground levels $|b\rangle$ and $|a\rangle$ and excited level $|c\rangle$ initially prepared in level $|b\rangle$. A weak write pulse nearly resonant on the $|b\rangle \leftrightarrow|c\rangle$ transition impinges on the ensemble. This write field induces Raman scattering of signal photons nearly resonant on the $|a\rangle \leftrightarrow|c\rangle$ transition with a temporal envelope identical to that of the write pulse [10]. Independently of the time at which a signal photon might be emitted, the Raman scattering imprints an idler excitation onto a unique spatial spin-wave mode [10]. After the write process is complete, the state of the idler spin wave can then be mapped to an idler field mode through application of a read field resonant on the $|a\rangle \leftrightarrow|c\rangle$ transition. The scattering dynamics are thus equivalent to those of a two-mode parametric amplifier, i.e., the signal-idler amplitude remains separable. Furthermore, by extending the write process over a long period $(0.8 \mu \mathrm{s})$, we are able to ensure that the photodetectors employed have a temporal resolution much shorter than the emitted signal field.

A remaining challenge here is to achieve the required high detection efficiency. By optimizing the write/read and signal/idler spatial modes, we obtain a measured signal detection efficiency of 0.17 , which may be compared with 0.08 in Ref. [11]. Ideally, the retrieval efficiency should be independent of storage time over the duration of an experimental trial. To achieve this we must compensate ambient magnetic fields which induce dephasing of the spin waves due to Larmor precession. Spin wave lifetimes of several milliseconds have been demonstrated by employing magnetically insensitive coherences and optical pumping [12,13]. This technique cannot be used here, however, since it would involve the application of a bias field resulting in oscillations of the retrieval efficiency as a function of storage time [12]. We therefore obtain sufficiently long memory lifetimes $(25 \mu \mathrm{s})$ by minimizing the ambient magnetic fields.

We consider a source of correlated signal and idler fields with the assumption that the two-photon amplitude is separable: $f\left(t, t^{\prime}\right)=\varphi_{s}(t) \varphi_{i}\left(t^{\prime}\right)$. We define the single-mode signal and idler annihilation operators $\hat{a}_{s, i} \equiv \int_{-\infty}^{\infty} d t \varphi_{s, i}^{*}(t) \hat{\psi}_{s, i}(t)$. The state produced by the effective two-mode parametric amplification process of the write-read process is given by [7]

$$
|\Psi(\chi)\rangle=\frac{1}{\cosh (\chi)} \sum_{n=0}^{2} \tanh ^{n} \chi \frac{\hat{a}_{s}^{\dagger n} \hat{a}_{i}^{\dagger n}}{n !}|v a c\rangle+O\left(\chi^{3}\right)
$$




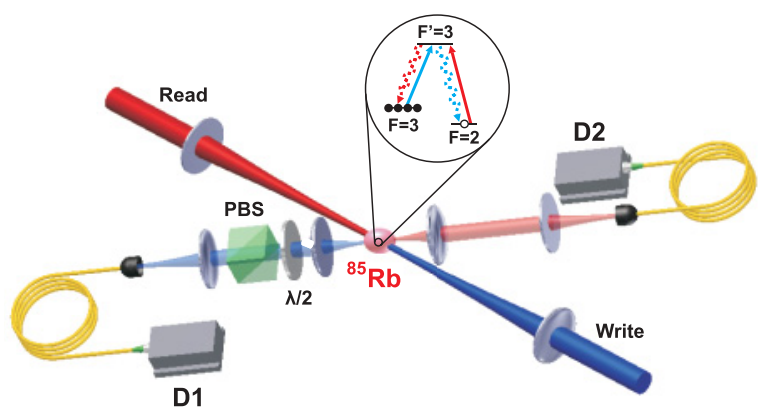

FIG. 1. (Color online) Schematic of experimental setup with the inset showing the atomic level scheme. Here, $\lambda / 2$ and PBS are half-wave plate and polarizing beam-splitter for the signal field, respectively, and D1 and D2 are single-photon detectors. See text for a discussion.

where $\chi$ is the interaction parameter, and the higher-order terms $O\left(\chi^{3}\right)$ account for multiple signal mode excitations and emission into other temporal modes arriving at the detector. The signal field undergoes a continuous photon-counting measurement as it impinges on detector $D 1$ with efficiency $\epsilon_{s}$ (see Fig. 1). We model the photodetector as one of unit efficiency preceded by a beam splitter with intensity transmittance $\epsilon_{s}$. In the absence of a detection event in the interval $\left(-\infty, t_{c}\right)$, the vector $|\Psi(\chi)\rangle$ is projected into the subspace in which no photons arrive at the hypothetical unit efficiency detector before the conditioning time $t_{c}$. We calculate the effects of the conditioning by applying the projection operator: $\exp \left(-\int_{-\infty}^{t_{c}} d t \hat{\psi}_{D 1}^{\dagger}(t) \hat{\psi}_{D 1}(t)\right)$ : to $|\Psi(\chi)\rangle$. Here, $\hat{\psi}_{D 1}(t) \equiv \sqrt{\epsilon_{s}} \hat{\psi}_{s}(t)+\sqrt{1-\epsilon_{s}} \hat{\xi}_{1}(t)$ and $\hat{\xi}_{1}(t)$ is a bosonic noise operator associated with detector $D 1$. This projection thus yields the conditioned state

$$
\begin{aligned}
& \sqrt{\pi_{0}\left(t_{c}\right)}\left|\Psi_{c}\left(t_{c}\right)\right\rangle \\
& =O\left(\chi^{3}\right)+\sum_{n=0}^{\infty} \frac{\left[\sqrt{1-\epsilon_{s} \mu\left(t_{c}\right)} \tanh (\chi)\right]^{n}}{\cosh \chi} \frac{\hat{c}^{\dagger n}\left(t_{c}\right)}{\sqrt{n !}} \frac{\hat{a}_{i}^{\dagger n}}{\sqrt{n !}}|\mathrm{vac}\rangle,
\end{aligned}
$$

where the increasing function $\mu\left(t_{c}\right) \equiv \int_{-\infty}^{t_{c}} d t\left|\varphi_{s}(t)\right|^{2}$, with $\mu(\infty)=1$. Provided the detection efficiency $\epsilon_{s}$ is not negligibly small, conditioning effectively results in a state with a reduced interaction parameter, where the signal mode $\hat{a}_{s}$ has been replaced by the conditioned bosonic mode

$$
\hat{c}\left(t_{c}\right)=-\sqrt{\frac{\mu\left(t_{c}\right)\left(1-\epsilon_{s}\right)}{1-\epsilon_{s} \mu\left(t_{c}\right)}} \hat{V}\left(t_{c}\right)+\sqrt{\frac{1-\mu\left(t_{c}\right)}{1-\epsilon_{s} \mu\left(t_{c}\right)}} \hat{\alpha}\left(t_{c}\right),
$$

in which the prior signal field, that arriving before $t_{c}$, is replaced by the vacuum noise field $\hat{V}\left(t_{c}\right)$, and the postsignal field, arriving after $t_{c}$, is defined by $\hat{\alpha}\left(t_{c}\right)=$ $\int_{t_{c}}^{\infty} d t \varphi_{s}^{*}(t) \hat{\psi}_{s}(t) / \sqrt{1-\mu\left(t_{c}\right)}$.

Taking the norm of Eq. (2), we find the probability for no photoelectric event registered before $t_{c}$

$$
\pi_{0}\left(t_{c}\right)=\frac{1}{1+\epsilon_{s} \mu\left(t_{c}\right) \sinh ^{2} \chi}+O\left(\chi^{3}\right) .
$$

Conditioned on the absence of a signal event in the interval $\left(-\infty, t_{c}\right)$, we can calculate the probability of registering at least one photoelectric detection event in the idler channel by taking the expectation value of the projection operator $\hat{P}_{i} \equiv: 1-\exp \left(-\hat{d}_{i}^{\dagger} \hat{d}_{i}\right):$ with respect to the conditioned state of Eq. (2), where $\hat{d}_{i}=\sqrt{\epsilon}_{i} \hat{a}_{i}+\sqrt{1-\epsilon_{i}} \hat{\xi}_{i}, \epsilon_{i}$ is the efficiency of the idler detector, and $\hat{\xi}_{i}$ is an idler noise operator. The second term of the operator $\hat{P}_{i}$ projects onto the subspace in which no idler photons are detected. Similarly, one finds the probability of detecting a signal between times $t_{1}$ and $t_{2}, p_{s \mid 0}\left(t_{1}, t_{2} ; t_{c}\right)$, corresponding to the expectation value of $\hat{P}_{s}\left(t_{1}, t_{2}\right)=: 1-\exp \left(-\int_{t_{1}}^{t_{2}} d t \hat{\psi}_{D 1}^{\dagger}(t) \hat{\psi}_{D 1}(t)\right):$. Finally, the joint conditioned probability that a photoelectric event is registered at both the idler detector (at any time during the trial) and the signal detector between times $t_{1}$ and $t_{2}$ corresponds to the expectation value of $\hat{P}_{i} \hat{P}_{s}\left(t_{1}, t_{2}\right)$. Dark counts on the signal and idler channels can be modelled by taking the noise fields associated with $\hat{\xi}_{s}(t)$ and $\hat{\xi}_{i}$ to be in coherent states; the dark count rates would be given by $B_{i}=\left\langle\hat{\xi}_{i}^{\dagger} \hat{\xi}_{i}\right\rangle$ and $B_{s}\left(t_{1}, t_{2}\right)=\int_{t_{1}}^{t_{2}} d t\left\langle\hat{\xi}_{s}^{\dagger}(t) \hat{\xi}_{s}(t)\right\rangle$. In the weak excitation limit where $B_{i} \sim B_{s}\left(t_{1}, t_{2}\right) \sim \sinh ^{2} \chi \ll 1$, these conditioned detection probabilities are, to first order in $\sinh ^{2} \chi$,

$$
\begin{aligned}
p_{s \mid 0}\left(t_{1}, t_{2} ; t_{c}\right) \approx & \epsilon_{s}\left[\mu\left(t_{2}\right)-\mu\left(t_{1}\right)\right] \sinh ^{2} \chi \\
& +B_{s}\left(t_{1}, t_{2}\right), \\
p_{i \mid 0}\left(t_{c}\right) \approx & \epsilon_{i}\left[1-\epsilon_{s} \mu\left(t_{c}\right)\right] \sinh ^{2} \chi+B_{i}, \\
p_{s i \mid 0}\left(t_{1}, t_{2} ; t_{c}\right) \approx & \epsilon_{s} \epsilon_{i}\left[\mu\left(t_{2}\right)-\mu\left(t_{1}\right)\right] \sinh ^{2} \chi .
\end{aligned}
$$

As intuitively expected, the conditioned idler probability, $p_{i \mid 0}\left(t_{c}\right)$, becomes progressively smaller as the conditioning interval increases. By contrast, the probabilities $p_{s \mid 0}\left(t_{1}, t_{2} ; t_{c}\right)$ and $p_{s i \mid 0}\left(t_{1}, t_{2} ; t_{c}\right)$ are proportional to the detection window $\mu\left(t_{2}\right)-\mu\left(t_{1}\right)$ and are identical to the corresponding unconditioned probabilities. The conditioning only manifests itself in $p_{s \mid 0}\left(t_{1}, t_{2} ; t_{c}\right)$ and $p_{s i \mid 0}\left(t_{1}, t_{2} ; t_{c}\right)$ through the requirement that the detection window occurs after $t_{c}$ and $\mu\left(t_{2}\right)-\mu\left(t_{1}\right) \leqslant 1-$ $\mu\left(t_{c}\right)$. From Eqs. (5) we can also determine the unconditioned, integrated probabilities $\left(t_{c}, t_{1} \rightarrow-\infty\right),\left(t_{2} \rightarrow \infty\right)$ [11]:

$$
\begin{gathered}
p_{s} \approx \epsilon_{s} \sinh ^{2} \chi+B_{s}, \\
p_{i} \approx \epsilon_{i} \sinh ^{2} \chi+B_{i}, \\
p_{s i} \approx \epsilon_{s} \epsilon_{i} \sinh ^{2} \chi .
\end{gathered}
$$

From these detection probabilities, one can define the experimentally measurable effective signal efficiency as

$$
\epsilon_{s}^{\prime} \equiv p_{s i} / p_{i}=\epsilon_{s}\left(1-\frac{B_{i}}{\epsilon_{i} \sinh ^{2} \chi+B_{i}}\right),
$$

which is reduced by background idler counts $B_{i}$. Inspection of Eqs. (5) and (7) reveals that this efficiency can also be obtained through the conditioned idler detection probability as

$$
\epsilon_{s}^{\prime}=-\frac{1}{p_{i}} \frac{d p_{i \mid 0}\left(t_{c}\right)}{d \mu} .
$$

This correspondence between the predictions of conditioned and unconditioned quantum dynamics provides a quantitative measure to experimentally test the dynamics of conditional quantum measurement.

To implement such a test, we prepare an optically thick atomic cloud of ${ }^{85} \mathrm{Rb}$ by switching on a magneto-optical trap (MOT) for a period of $14 \mathrm{~ms}$ (Fig. 1). The atomic ground levels 
$\{|a\rangle ;|b\rangle\}$ correspond to the $5 S_{1 / 2}, F_{a, b}=\{2,3\}$ hyperfine levels, while the excited level $|c\rangle$ represents the $5 P_{1 / 2}, F_{c}=3$ level of the $D_{1}$ line at $795 \mathrm{~nm}$. After switching off the MOT fields, the experimental sequence begins with all of the atoms pumped into level $|b\rangle$ by sequentially switching off first the trapping light followed $10 \mu$ s later by the repumping light. The quadrupole magnetic field of the MOT is extinguished for the $2.5 \mathrm{~ms}$ duration of the measurement sequence. Compensation of the ambient magnetic field is provided by three pairs of Helmholtz coils.

The measurement sequence consists of 1666 cycles of duration $1.5 \mu \mathrm{s}$. The cycle begins when a weak, approximately square, linearly polarized write laser pulse, tuned to the $|b\rangle \leftrightarrow|c\rangle$ transition, illuminates the ensemble for $T=$ $0.8 \mu \mathrm{s}$; that is, in each cycle $\mu\left(t_{c}=0\right)=0$ and $\mu(T)=1$. The light pulse generates an orthogonally polarized signal field by spontaneous Raman scattering on the $|c\rangle \leftrightarrow|a\rangle$ transition together with spin wave excitation of the atomic medium associated with the $|b\rangle \leftrightarrow|a\rangle$ hyperfine coherence [14]. After a 200 ns delay, a 200 ns long read pulse, tuned to the $|a\rangle \leftrightarrow|c\rangle$ transition, illuminates the atoms. This read field, with power $170 \mu \mathrm{W}$ and linear polarization orthogonal to that of the write pulse, converts the atomic spin excitation into an orthogonally polarized idler field, which is emitted on the $|c\rangle \leftrightarrow|b\rangle$ transition. Both the write-read and signal-idler pairs of mode-matched fields are counterpropagating, with Gaussian waists of $400 \mu \mathrm{m}$ for the former and $130 \mu \mathrm{m}$ for the latter. The signal and idler fields are measured by single photon detectors D1 and D2, respectively.

The photoelectric detection events for the signal and idler fields are measured and recorded with 2 ns time resolution, allowing conditioned and unconditioned detection probabilities to be determined. The unconditioned detection probability for the idler field is defined by the ratio of the number of cycles, $N_{i}$, with at least one photoelectric detection event recorded to the total number of cycles: $P_{i} \equiv N_{i} / N_{T}$. The conditioned probability is determined similarly, except that all cycles in which a signal photoelectric event has been recorded prior to time $t_{c}$ are omitted, hence $P_{i \mid 0}\left(t_{c}\right) \equiv N_{i \mid 0}\left(t_{c}\right) / N_{T}$. In order to test the predictions of the conditional quantum theory, we measure both the unconditioned signal-idler coincidence probability, $P_{s i} \equiv N_{s i} / N_{T}$, and the gradient of the conditional idler detection probability, $D_{i \mid 0}\left(t_{c}\right) \equiv-d P_{i \mid 0} / d \mu\left(t_{c}\right)$, as a function of $\mu\left(t_{c}\right)$, which according to Eq. (8), must be equal to $\epsilon_{s}^{\prime} P_{i}$. By varying $\epsilon_{s}$ using the half-wave plate placed in the signal beam path before the polarizer (Fig. 1), we measure a set of values for $P_{s i}$. For each plate setting, we construct $D_{i \mid 0}\left(t_{c}\right)$

$$
D_{i \mid 0}\left(t_{c}\right) \simeq \frac{P_{i \mid 0}\left(t_{c}\right)-P_{i \mid 0}\left(t_{c}+\Delta t\right)}{\mu\left(t_{c}+\Delta t\right)-\mu\left(t_{c}\right)},
$$

where $\Delta t$ is a sufficiently small time interval that the determined $D_{i \mid 0}\left(t_{c}\right)$ does not depend on its value. The results are presented in Fig. 2 and show very good agreement between the conditional measurement data, $D_{i \mid 0}\left(t_{c}\right) / P_{i}$, and the unconditional data, $P_{s i} / P_{i}$, which Eqs. (7) and (8) predict should be equal. The error bars on conditional data are based on the statistics of the photoelectric counting events, while statistical errors on the unconditioned data are negligible.

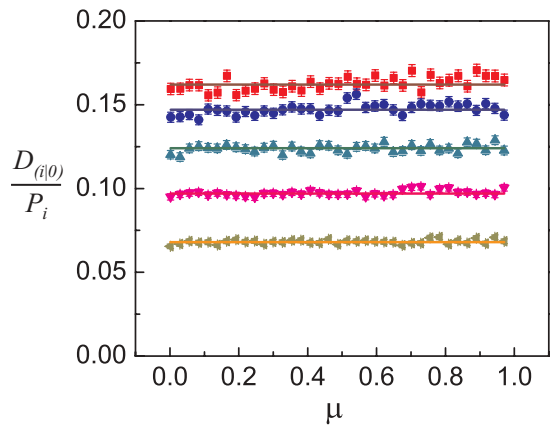

FIG. 2. (Color online) Experimental data for $D_{i \mid 0}\left(t_{c}\right) / P_{i}$ (discrete data) and $P_{s i} / P_{i}$ (solid lines) vs. signal field gate function $\mu\left(t_{c}\right)$ for five different settings of the half-wave plate (Fig. 1). According to conditional quantum theory, these should be equal, Eqs. (7) and (8). Data shown from top to bottom correspond to decreasing measured signal efficiencies. The data acquisition time was $5 \mathrm{~h}$.

At high measured efficiencies, there is a small systematic discrepancy in excess of the statistical errors that requires a careful examination in order to exclude spurious effects which could potentially mimic the predictions of conditional quantum theory. The two most relevant processes are (1) the residual effects of Raman scattering of the write pulse and (2) the Larmor precession of the hyperfine coherences in the uncompensated magnetic field. Although Raman scattering is responsible for the creation of the signal photon and the accompanying spin excitation, excessive scattering into undetected field modes can slightly deplete the population of level $|b\rangle$ during the write process and therefore reduce the efficiency with which spin waves generated early in the write process are retrieved. We can model this effect using the timedependent retrieval efficiency $\eta(1-\mu)=\epsilon_{i} \exp [-\alpha(1-\mu)]$, where $1-\mu$ is proportional to the time a spin wave is exposed to the deleterious effects of the write beam. This would manifest itself in an increasing time-dependent correction to $\epsilon_{s}^{\prime}$ in Eq. (8) and as a reduced joint signal-idler coincidence detection probability at small values of $\mu$. Based on this picture, we can model $p_{s i}(\mu, \mu+\Delta \mu) / \Delta \mu \sim p_{s} \exp (-\alpha(1-\mu))$, where $\alpha$ is proportional to the write pulse energy, as shown by the data in Fig. 3. As can be seen from Fig. 3, for the $0.1 \mu \mathrm{W}$ power at which data in Fig. 2 were taken, these effects are not significant. Similarly, the Larmor precession reduces $p_{s i}$ for small values

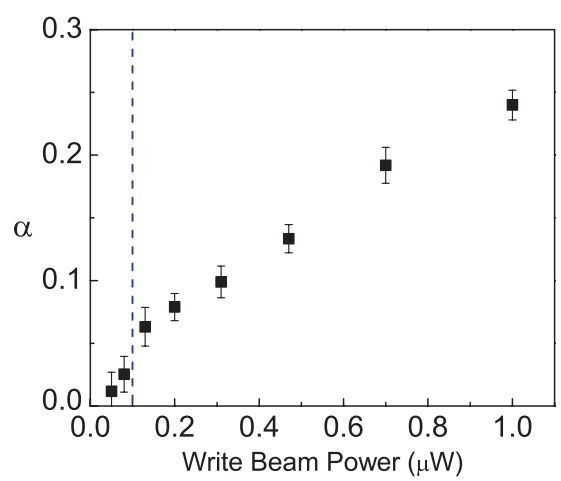

FIG. 3. (Color online) Coefficient $\alpha$ as the function of write beam power (see text). The vertical dashed line shows the value of write beam power at which the data in Fig. 2 were taken. 
of $\mu\left(t_{c}\right)$, according to $p_{s i} \sim \exp \left(-\gamma(1-\mu)^{2}\right)$ [15,16]. For the measured coherence time, $\tau_{c} \approx 25 \mu \mathrm{s}$, and maximum write-read delay, $T_{t} \sim 1 \mu \mathrm{s}$, the expected decoherence effect is negligible $\left(\gamma=\left(T_{t} / \tau_{c}\right)^{2} \approx 0.004\right)$.
In summary, we have observed conditional dynamics of a correlated atomic spin wave-light system. This work was supported by the National Science Foundation and the Air Force Office of Scientific Research.
[1] H. J. Carmichael, An Open Systems Approach to Quantum Optics (Springer, Berlin, 1993).

[2] C. W. Gardiner and P. Zoller, Quantum Noise: A Handbook of Markovian and Non-Markovian Quantum Stochastic Methods with Applications to Quantum Optics, 2nd ed. (Springer-Verlag, Berlin, 2000).

[3] G. T. Foster, L. A. Orozco, H. M. Castro-Beltran, and H. J. Carmichael, Phys. Rev. Lett. 85, 3149 (2000).

[4] W. P. Smith, J. E. Reiner, L. A. Orozco, S. Kuhr, and H. M. Wiseman, Phys. Rev. Lett. 89, 133601 (2002).

[5] H. J. Carmichael et al., in Prog. Opt. 46, 355 (2004).

[6] N. Katz et al., Science 312, 1498 (2006).

[7] D. F. Walls and G. J. Milburn, Quantum Optics (Springer-Verlag, Berlin, 1994).
[8] D. Branning, W. P. Grice, R. Erdmann, and I. A. Walmsley, Phys. Rev. Lett. 83, 955 (1999).

[9] C. K. Law, I. A. Walmsley, and J. H. Eberly, Phys. Rev. Lett. 84, 5304 (2000).

[10] S. D. Jenkins, Ph.D. thesis, Georgia Institute of Technology, 2006.

[11] D. N. Matsukevich et al., Phys. Rev. Lett. 97, 013601 (2006).

[12] R. Zhao et al., Nature Physics 5, 100 (2009).

[13] Y. O. Dudin et al., Phys. Rev. Lett. 103, 020505 (2009).

[14] L.-M. Duan, M. D. Lukin, J. I. Cirac, and P. Zoller, Nature 414, 413 (2001).

[15] D. N. Matsukevich et al., Phys. Rev. Lett. 96, 033601 (2006).

[16] S. D. Jenkins, D. N. Matsukevich, T. Chaneliere, A. Kuzmich, and T. A. B. Kennedy, Phys. Rev. A 73, 021803(R) (2006). 